\title{
Application of fuzzy logic to determine the odour intensity of model gas mixtures using electronic nose
}

\author{
Bartosz Szulczyński ${ }^{1, *}$, Jacek Gębicki ${ }^{1}$, and Jacek Namieśnik ${ }^{2}$ \\ ${ }^{1}$ Department of Chemical and Process Engineering, Faculty of Chemistry, Gdańsk University of \\ Technology, 11/12 Gabriela Narutowicza Street, 80-233 Gdańsk, Poland \\ ${ }^{2}$ Department of Analytical Chemistry, Faculty of Chemistry, Gdańsk University of Technology, 11/12 \\ Gabriela Narutowicza Street, 80-233 Gdańsk, Poland
}

\begin{abstract}
The paper presents the possibility of application of fuzzy logic to determine the odour intensity of model, ternary gas mixtures ( $\alpha$-pinene, toluene and triethylamine) using electronic nose prototype. The results obtained using fuzzy logic algorithms were compared with the values obtained using multiple linear regression (MLR) model and sensory analysis. As the results of the studies, it was found the electronic nose prototype along with the fuzzy logic pattern recognition system can be successfully used to estimate the odour intensity of tested gas mixtures. The correctness of the results obtained using fuzzy logic was equal to $68 \%$.
\end{abstract}

\section{Introduction}

Public opinion on the state of the environment depends on many factors, among which the smell of air we breathe occupies one of the important factors. Today, many areas of human activity, in particular waste water treatment and municipal waste management, are a source of odour nuisances [1,2]. For this reason, measurement and monitoring of odour nuisance is an extremely important issue to be addressed. According to EN 13725:2003, the odour concentration is determined by the dynamic olfactometry method. From a practical point of view, it would be most valuable to directly determine the odour nuisances using the results of analytical air monitoring. The solution to this problem would keep the public about odour air quality. In addition, it will be possible to forecast the extent of significant nuisance of the projected objects based on the results of simulation of odour spread in the environment of emitters.

One of the parameters that characterize the smell is an odour intensity. It is one of the most commonly determined odour parameters. It is defined as the perceived strength of odour sensation that will be triggered by a specific stimulus [3]. Quantitative odour intensity determinations are typically based on sensory analysis in which a sensory panel determines the intensity of the odour using a verbal point scale. An example of such a scale

${ }^{*}$ Corresponding author: bartosz.szulczynski@.pg.edu.pl 
is the seven-point scale described in German Standard VDI 3940. Degrees corresponding to the respective odour intensity are shown in Table 1.

Table 1. Odour intensity scale described in German Standard VDI 3940.

\begin{tabular}{|c|c|}
\hline Odour strength & Intensitylevel \\
\hline Not perceptible & 0 \\
\hline Very weak & 1 \\
\hline Weak & 2 \\
\hline Distinct & 3 \\
\hline Strong & 4 \\
\hline Very strong & 5 \\
\hline Extremely strong & 6 \\
\hline
\end{tabular}

The paper presents the possibility of fuzzy logic application to determine the odour intensity of model ternary gas mixtures using electronic nose prototype. As the ingredients of the mixtures three substances were selected: $\alpha$-pinene, toluene and triethylamine. These substances have low odour thresholds and have been identified in the air nearby waste water treatment plant or landfill [4-6]. The results obtained using fuzzy logic algorithms were compared with the values obtained using multiple linear regression model and sensory analysis.

\section{Electronic nose}

In recent years there has been a significant increase in interest in the use of artificial senses in chemical analysis. Particular position is occupied by devices that mimic the sense of human smell - electronic noses. These instruments allow a holistic analysis of the gas mixture composition, without separating it into individual components (as is the case with the use of chromatographic methods) [7]. In terms of construction, they are equipped with a set of several chemical gas sensors. The commercially available volatile organic compounds sensors are the most commonly used sensor types $[8,9]$.

E-noses in their functioning resemble the sense of human smell (Figure 1). Sensors are the analogs of the olfactory receptors. They turning the chemical information into an analytically useful signal. Then the signal is sent to the recognition system, which in the case of human is the brain, and in the case of e-nose is the appropriate mathematical algorithm. The most commonly used data processing methods are: principal component analysis (PCA), principal component regression (PCR), partial least square regression (PLS), fuzzy logic and artificial neural networks (ANN) [10-14]. 


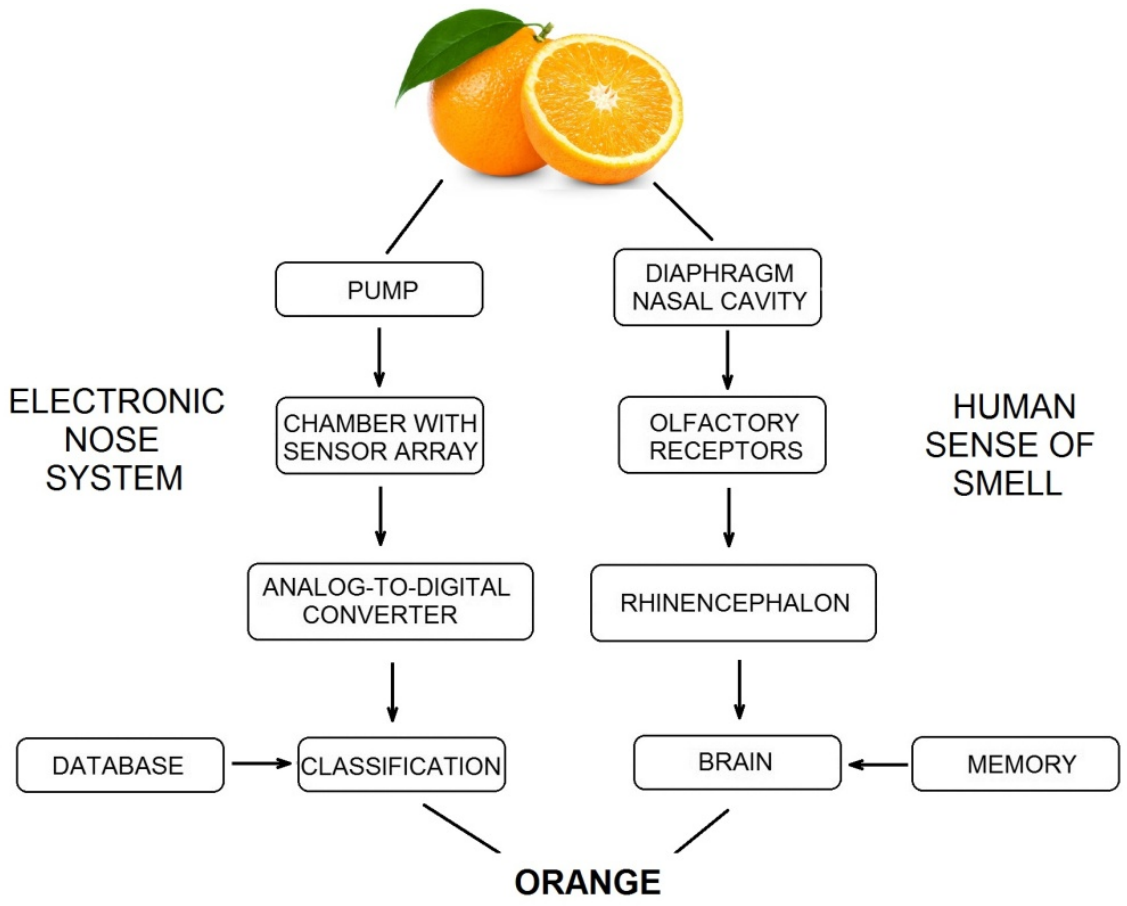

Fig. 1. Diagram of the electronic nose operation along with human sense of smell.

The electronic nose consists of four basic and independent elements:

- sampling system - provide reproducible and stable measurement conditions (gas flow velocity, humidity, temperature) and eliminates all undesirable factors that can affect the sensor response,

- detection system - a set of chemical sensors located in the measuring chamber which exhibit different selectivity and sensitivity to the individual components of the sample, but as a whole they generate a characteristic chemical pattern of the gas mixture (so-called "fingerprint"),

- data processing system - responsible for signal processing,

- pattern recognition system - assigns the received set of signals to one of the pattern classes.

The most commonly used type of sensors in electronic noses are Metal Oxide Sensors (MOS). The sensor signal's dependence on the concentration of the gaseous substance in the sensor environment is described by the formula (1):

$$
R_{\mathrm{s}}=B \cdot c^{-\alpha}
$$

where: $R_{s}$ - sensor resistance; $B, \alpha$ - constant values characterizing the sensor; $c-$ substance odour concentration.

Previous studies have shown that the theoretical dependence (2) is linear [8]. For this reason, a multiple linear regression model (3) was proposed combining the odour intensity with the electronic nose sensor signals:

$$
\begin{gathered}
I=\mathrm{f}(\log S) \\
I=a_{0}+a_{1} \prod \operatorname{tog} S_{1}+a_{2} \operatorname{Tog}_{2} S_{2}+\ldots+a_{n} \operatorname{\prod og} S_{n}
\end{gathered}
$$


where: $I$ - odour intensity; $S$ - sensor signal; $a$ - model parameter, $n$ - number of sensors working in electronic nose system.

\section{Fuzzy logic}

One of the most interesting approaches in the field of e-nose data analysis is fuzzy logic. Classical logic system is based on the two values, mostly represented by 0 and 1 , or true and false. The boundary between them is unambiguously defined and unchanging. Fuzzy logic is an extension of the classical approach to approach closer to human brain - it introduces additional values between standard 0 and 1 . Blurring the boundaries between them gives the opportunity to come up with values between this interval (eg, almost false, half truth). An example of fuzzy and non-fuzzy sets is shown in Figure 2.
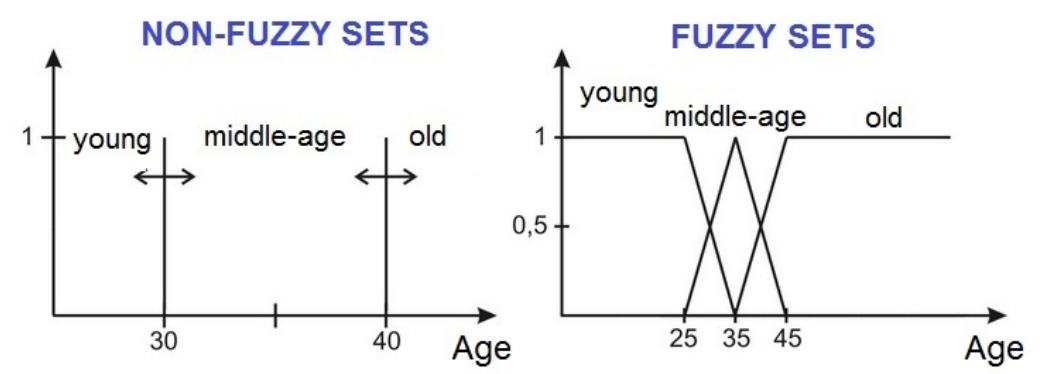

Fig.2. An example of fuzzy and non-fuzzy sets.

Fuzzy logic allows a fuzzy description of real systems and is an alternative to describing systems using classical binary logic. The basis of fuzzy sets theory was introduced by L. Zadech. From this point on, the methods developed on the basis of the theory are of great interest. Their development goes hand in hand with the growing number of applications of fuzzy logic in practice. The proposed scheme of using fuzzy logic to estimate the odour intensity is presented in the Figure 3.

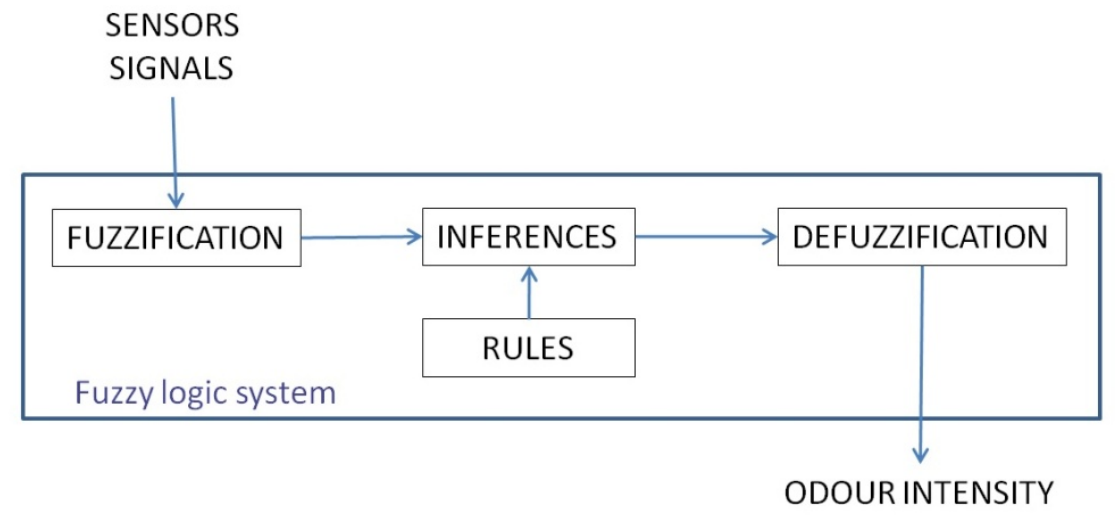

Fig.3. Proposed scheme of using fuzzy logic to estimate the odour intensity using electronic nose.

In the case of non-fuzzy sets, the membership function is rectangular. It is set to 0 (no membership in the set) or one (membership in the set). In case of fuzzy sets, other membership functions, such as trapezoid, triangular, gaussian, sigmoidal are used. In this 
work, trapezoid functions were used. In the Figure 4, the defined fuzzy set for the e-nose sensor signal value is shown as an example. The chosen values were selected using optimization methods.

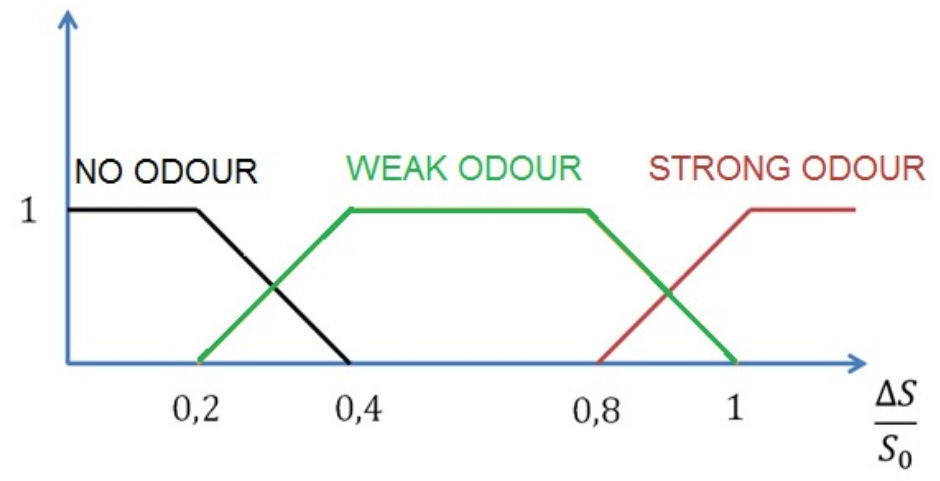

Fig.4. An example of fuzzy and non-fuzzy sets.

For the fuzzy system's output variable, seven fuzzy sets defined by the names in Table 1 were created. The output value is calculated by the fuzzy system, using a set of rules, based on the input values. Thus, the determined odour intensity corresponds to a specific set of sensor signal values. In the application process, a set of rules based on conjunctive operations is applied. An example of the rule is shown in formula 4:

$$
\text { IF }\left(S _ { l } \in N O \text { ODOUR) AND ... AND } \left(S_{8} \in\right.\right. \text { NO ODOUR) THEN OIE(Not perceptible) }
$$

Fuzzy inference process in the developed model proceeds in three stages (Figure 3). At the entrance of the model are introduced eight input variables (each sensor signals). In the fuzzification block, the degree of belonging of individual values to fuzzy sets is calculated.In the next block, the resulting function of the model output is calculated. In this process, the rule base is used. At the defuzzification stage, the resulting affinity function is the basis for calculating the value of the output variable. The center of gravity mechanism was used for this purpose.

\section{Experimental}

In order to determine the usefulness of the fuzzy logic to determine the odour intensity using the electronic nose, 22 aqueous solutions were made. The samples 1,6 and 11 were prepared in such a way that their odour intensity was equal to 1 according to the VDI 3940 standard. Five aqueous solutions characterized by a 2-step dilution were prepared for each of the investigated substances. Prepared solutions were used for determination of odour intensity of each sample, which was performed by a team of assesso. Obtained results were utilized to determine the dependency of odour intensity versus logarithm of concentration of particular odorous substance in water (according to the Weber-Fechner law). These dependencies were used to determine concentration of particular substances in deionised water corresponding to odour intensity equal to 1 . These concentrations were respectively: $0.6 \mathrm{ppm} \mathrm{v/v}$ toluene, $0.1 \mathrm{ppm} \mathrm{v/v} \alpha$-pinene and $1.5 \mathrm{ppm} \mathrm{v/v}$ triethylamine. Other samples were prepared in appropriate volume proportions defined by the position of the sample in the ternary system (Figure 5). 


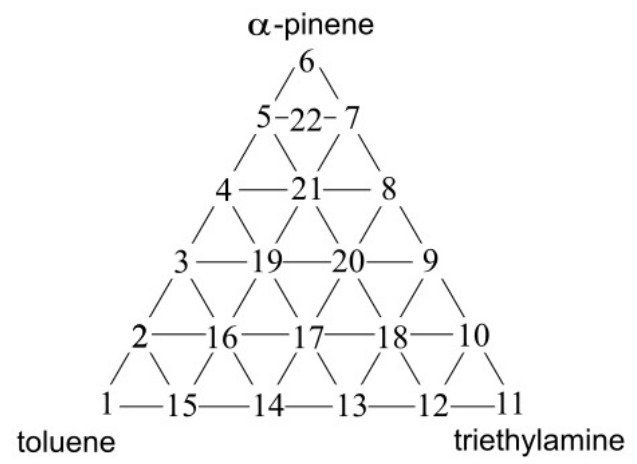

Fig.5. A ternary plot illustrating the composition of the tested mixtures.

The odour intensity of these samples was determined in three ways:

1. using a sensory panel (sensory analysis) according to the procedure described in [15],

2. using electronic nose and fuzzy logic,

3. using electronic nose and multiple linear regression model (3). Model parameters and their statistical significance are presented in Table 2. The model was used for further calculations after removing non-significant members. The final form is represented by the equation (5) - the determination coefficient of the model was equal to 0.72 :

$$
I=-8.45-6.35 \log S_{1}+14.43 \operatorname{Tog} S_{4}
$$

Table 2. Calculated model parameters and their statistical significance.

\begin{tabular}{|c|c|c|}
\hline Coefficient & Calculated value & $\begin{array}{c}\text { Statistical } \\
\text { significance }\end{array}$ \\
\hline$a_{0}$ & -12.53 & yes \\
\hline$a_{1}$ & -3.22 & yes \\
\hline$a_{2}$ & 1.98 & no \\
\hline$a_{3}$ & -0.44 & no \\
\hline$a_{4}$ & 15.22 & yes \\
\hline$a_{5}$ & -2.55 & no \\
\hline$a_{6}$ & 0.44 & no \\
\hline$a_{7}$ & -0.39 & no \\
\hline$a_{8}$ & 2.55 & no \\
\hline
\end{tabular}

The analyzes were carried out using an electronic prototype equipped with a measuring chamber containing 8 MOS sensors manufactured by Figaro Engineering inc: TGS2104, TGS2106, TGS2180, TGS2600, TGS2602, TGS2201A, TGS2201B and TGS2611. The measurement system is shown in Figure 6. 


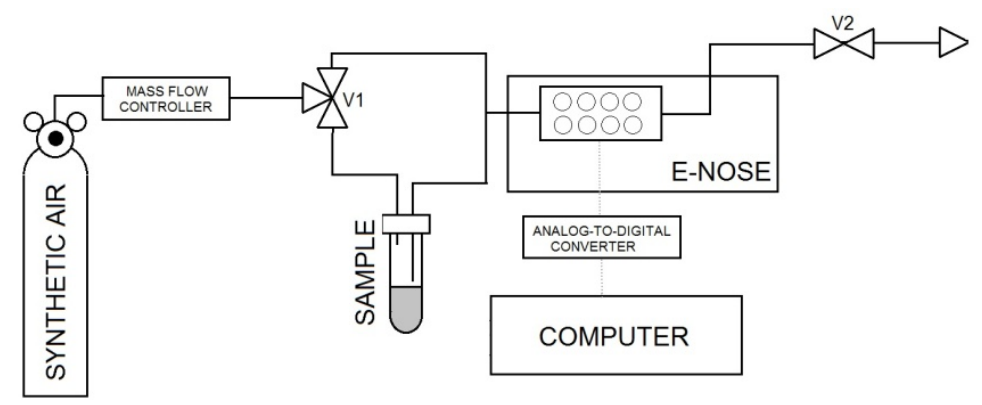

Fig.6. Electronic nose measurement system.

Synthetic air flow through a system at a constant flow rate of $330 \mathrm{~cm}^{3} \mathrm{~min}^{-1}$. It was controlled by a mass flow controller. The headspace analysis of prepared samples was conducted. By changing the position of the valve V1, the air flowed through the sample, which then passed to the measurement chamber. The electronic nose worked in the stopflow mode [16]: the sample flow time was 40 seconds and the stop time of the mixture in the sensors chamber - 20 seconds (after closing V2 valve). After this time the purified air was returned to the measurement chamber for regeneration of the sensors. Signals from the sensors were recorded using an ADC (Simex SIAi-8) and saved on the computer. Data analysis and other calculations were performed using RStudio Desktop (v. 1.0.143) software.

\section{Results and discussion}

In Figure 7 are presented statistical differences between the determined odour intensity values (in pairs: sensory analysis - fuzzy logic and sensory analysis - multiple linear regression).

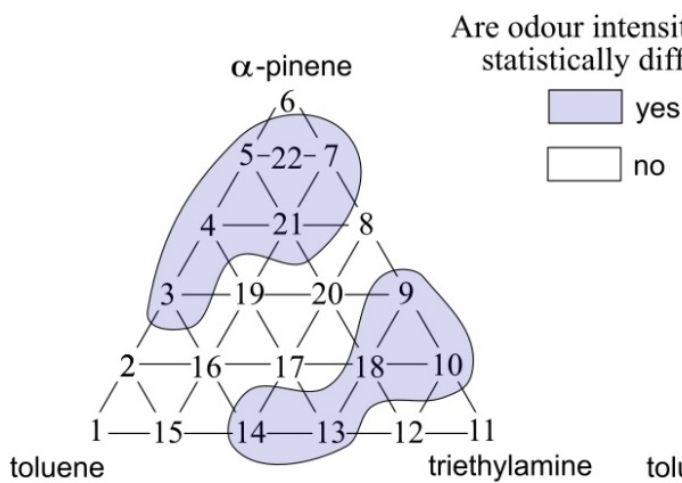

(a)
Are odour intensity values

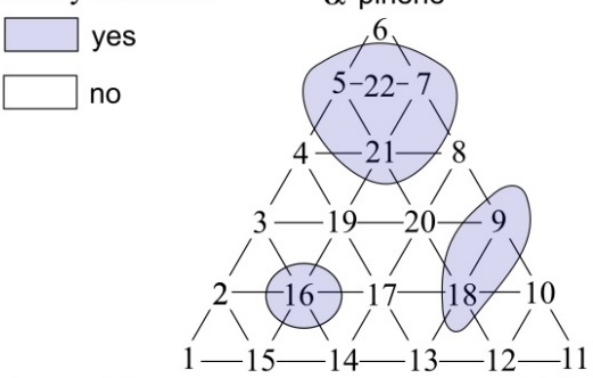

toluene

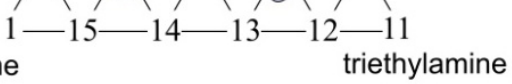

(b)

Fig.7. Ternary plots of gas mixtures odour intensity statistical differences: (a) determined by the multiple linear regression model, (b) fuzzy logic, compared to the values obtained by sensory analysis.

As the results of the studies, it was found that the electronic nose prototype along with the fuzzy logic pattern recognition system can be successfully used to estimate the odour intensity of $\alpha$-pinene, toluene and triethylamine as compounds potentially responsible for the presence of odour nuisances in the vicinity of the landfill or wastewater treatment plant. 
Determining the odour intensity using both: fuzzy logic and multiple linear regression model does not fully match the expected results (obtained by sensory analysis). Comparing the number of correct results showed that the correctness of results was better for the fuzzy logic model (68\%) compared to the multiple linear model (50\%). However, it is important to note that the creation of rules for fuzzy logic is much more complicated than the determination of linear model parameters and it requires some expert knowledge.

Observed differences in odour intensity determination may be due to the presence of odour interactions between the gas mixture components [12]. Conducted studies have shown that the greatest differences were observed for samples with high $\alpha$-pinene content. In this case, the discrepancy may be caused by masking of unpleasant odours by the nicely scented $\alpha$-pinene.

The investigations were financially supported by the Grant No. UMO-2015/19/B/ST4/02722 from the National Science Centre (Poland).

\section{References}

1. I. Sówka, Ł. Pachurka, Y. Bezyk, A. Grzelka, U. Miller, Ochrona Środowiska i Zasobów Naturalnych 28, 16-21 (2017)

2. I. Sówka, U. Miller, P. Sobczyński, Eviron. Prot. Eng. 42, 143-152 (2016)

3. J. Kośmider, B. Mazur-Chrzanowska, Odory (Wydawnictwo Naukowe PWN, Warszawa, 2012)

4. J.J. Fang, N. Yang, D.Y. Cen, L.M. Shao, P.J. He, Waste. Manage. 32, 1401-1410 (2012)

5. P. Lewkowska, B. Cieślik, T. Dymerski, P. Konieczka, J. Namieśnik, Environ. Res. 151, 573-589 (2016)

6. E. Davoli, M.L. Gangai, L. Morselli, D. Tonelli, Chemosphere 51, 357-368 (2003)

7. F. Rock, N. Barsan, U. Weimar, Chem. Rev. 108, 705-725 (2008)

8. B. Szulczyński, J. Gębicki, Environments 4, 21 (2017)

9. K. Arshak, E. Moore, G.M. Lyons, J. Harris, S. Clifford, Sensor Rev. 24, 181-198 (2004)

10. J. Gębicki, B. Szulczyński, M. Kamiński, Meas. Sci. Technol. 26, 125103 (2015)

11. W. Wojnowski, T. Majchrzak, T. Dymerski, J. Gębicki, J. Namieśnik, Meat Sci. 131, 119-131 (2017)

12. B. Szulczyński, J. Namieśnik, J. Gębicki, Sensors 17, 2380 (2017)

13. M. Badura, A. Szczurek, P.M. Szecówka, Sens. Actuators B Chem. 188, 815-823 (2013)

14. S.M. Scot, D. James, Z. Ali, Microchim. Acta 156, 183-207 (2006)

15. J. Gębicki, T. Dymerski, S. Rutkowski, Environ. Prot. Eng. 40, 103-116 (2014)

16. M. Maciejewska, A. Szczurek, L. Ochromowicz, Sens. Actuators B Chem. 141, 417423 (2009) 